\title{
Nano-Mechanics Reveal Resilience in Nacre of Mollusk Shells and Pearls
}

Jiseok Gim ${ }^{1}$, Noah Schnitzer ${ }^{2}$, Laura Otter ${ }^{3}$, Yuchi Cui ${ }^{4}$, Alden Koch $^{4}$, Sébastien Motreuil ${ }^{5}$, Frédéric Marin $^{5}$, Stephan Wolf ${ }^{6}$, Dorrit Jacob ${ }^{3}$, Amit Misra ${ }^{4}$ and Robert Hovden ${ }^{4}$

${ }^{1}$ Materials Science and Engineering, University of Michigan, Ann Arbor, Michigan, United States, ${ }^{2}$ Cornell University, Ithaca, New York, United States, ${ }^{3}$ Macquarie University, Sydney, New South Wales, Australia, ${ }^{4}$ University of Michigan, Ann Arbor, Michigan, United States, ${ }^{5}$ Université de Bourgogne Franche-Comté, Dijon, Bourgogne, France, ${ }^{6}$ Friedrich-Alexander-University Erlangen-Nürnberg, Erlangen, Bayern, Germany

The inherent tradeoff between strength and toughness inspires new design approaches to structural materials with high damage tolerance [1]. While plastic deformation degrades materials' strength and performance lifetime, it is the key attribute for toughness and resistance to fracture. Thus, optimizing mechanical properties for predictable and non-catastrophic failure motivates novel design of modern high-performance structural materials [2,3]. Among the diverse set of structural biominerals that straddle the gap between strength and toughness, nacre is the prototypical supermaterial [4-8]. Nacre forms in many mollusk shells and is the primary structure of lustrous pearls. In nacre of both pearls and shells, we show a nanostructure that optimizes structural resilience. The biomineralization precisely controls secretion of organic and inorganic ingredients and hierarchically designs the combination of soft nanoscale organic components with inorganic nanograins that govern the formation of pearls and shells. After crack initiation, bulk nacre shows a 40 -fold higher fracture toughness than the monolithic/single crystal calcium carbonate from which it is constructed [5, 9-13]. Thus, a central focus has been placed on understanding the principle mechanisms of nacre's nanoscale formation and mechanical properties to inspire new designs in next-generation high-performance structural materials [4-13]. However, nacre's ability to undergo limited deformation and dissipate critical stresses before fracture has not yet been quantified nor correlated with nanomechanical processes.

Our investigation of nacre deformation reveals the underlying nanomechanics that govern the structural resilience and absorption of mechanical energy [1]. High-resolution scanning / transmission electron microscopy (S/TEM) combined with in-situ nano-indentation has been adapted to biomineral systems to allow sub-nanometer resolution imaging of the nanomechanical deformation processes and provide precise assessment of when and where fracture occurs. On a system level, nacre can sustain several fractures before total failure due to its hierarchical soft-hard matter design. This means the yield stress of nacre is not typically defined by crack initiation. When fracture occurs, we show the organic components restrict crack propagation both within and between tablets, sustaining the overall macroscale architecture through multiple fractures to allow further structural loading (Fig. 1a-c). This allows nacre to absorb significantly greater mechanical energy than monolithic aragonite. This extends the damage tolerance of the superstructure beyond a single fracture.

In contrast, prismatic calcite and monolithic aragonite exhibit limited deformation before the yield stress is followed by catastrophic failure or crack runaway (Fig. 1d-i) from cone cracking at indentation. Monolithic aragonite responds to strain with stress contours radiating from the point of contact. Prismatic calcite from the Mediterranean fan mussel (Pinna Nobilis) — behaved similar to monolithic aragonite however indentation near an organic interface showed significant attenuation into an adjacent prism (Fig. 1e). When compared to monolithic calcite, we clearly see nacre's interlamellar membranes reshape compressive strain fields. Nacre's inorganic-organic architecture reliably absorbed $\sim 3$ times more mechanical energy than prismatic calcite and monolithic aragonite before total failure (Fig. 2a). At failure, the organic components in nacre impede crack propagation both within and between tablets (Fig. 1c, 2b-c). The smaller organic inclusions embedded within the inorganic matrix hinder crack propagation within the tablet and were observed to blunt and deflect cracks 
(Fig. 2c). The interlamellar membrane likewise hampers propagation between tablets, where cracks often terminate or jump at the interface (Fig. 2b).

The present in situ S/TEM nanoindentation study illuminates nacre's distinct non-linear elastic deformation processes that provide high resilience. We see how large forces can drive nacre into locked states that allow the material to distribute strain across tablets. Even after fracture, failure is mitigated through barriers to crack propagation that preserve the macroscale architecture and allow nacre to sustain continued loading without a noticeable change of structure in its mechanical response. This approach enables investigation of the wider range of evolutionary optimized biominerals to reveal advantages underlying each's nanomechanical design. The observed mechanisms reported in this work may guide theoretical models of deformation behavior, and the demonstration of in situ S/TEM nanoindentation of nacre opens the possibility of other in situ S/TEM such as bending, tensile, etc. across a wider range of biological and bio-inspired composites. For nacre under compression, the rich multiscale resilient deformation processes and interlamellar locking inspires new synthetic routes to complex structural materials.
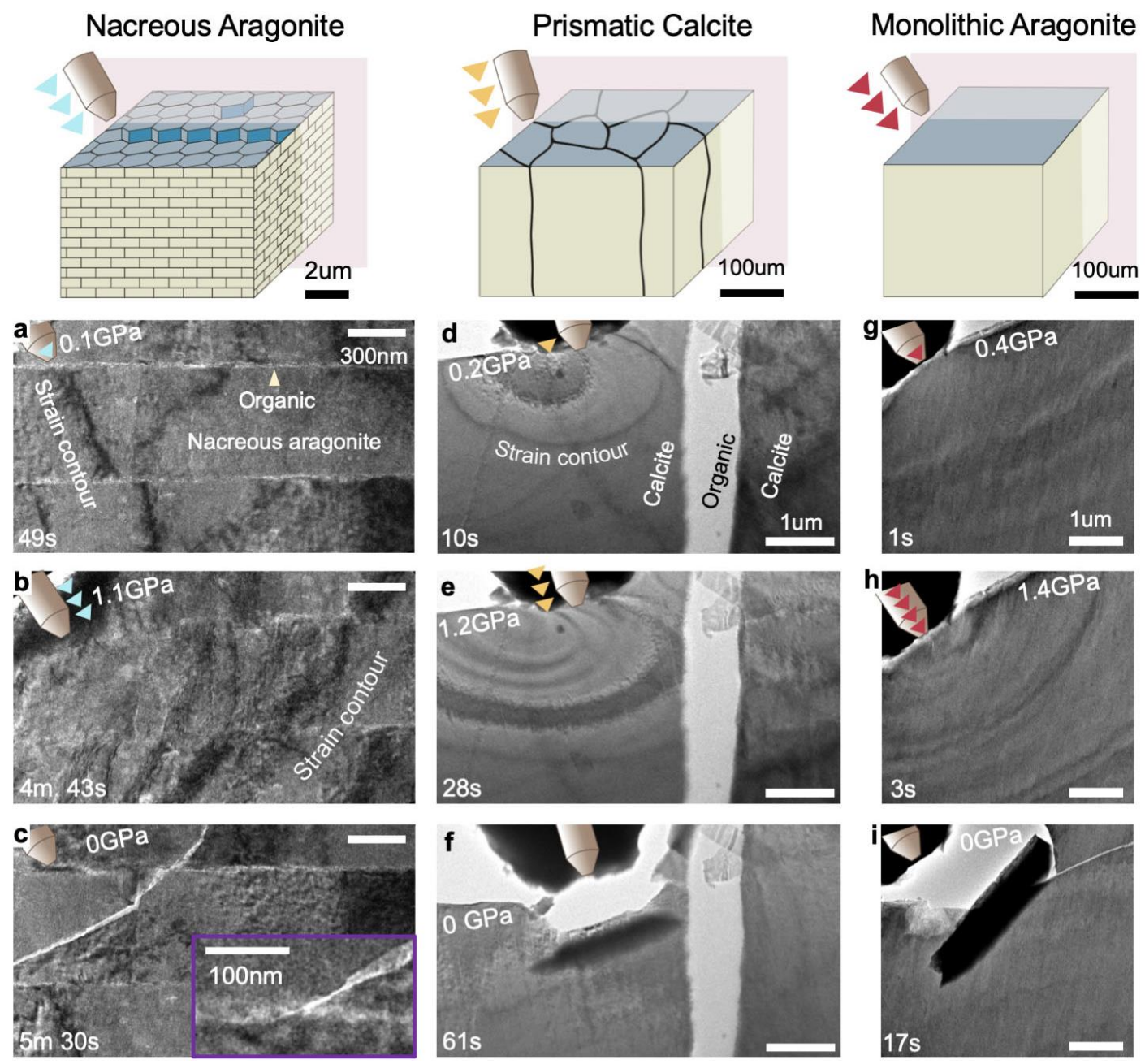

Figure 1. Figure 1. Toughening processes of nacre, prismatic calcite, and monolithic aragonite. Bright field TEM images of the cross-sectional a-c. nacreous region, d-f. prismatic calcite region, g-i. nonbiogenic, monolithic aragonite during in situ TEM indentation. The nacreous tablets made contact on the side of the cube corner tip (tip diameter: $\sim 100 \mathrm{~nm}$ ). The prismatic calcite region and monolithic aragonite 
made contact on the conospherical tip (tip diameter: $\sim 1 \mu \mathrm{m}$ ). Inset in (c) shows crack blunting at the organic interface.
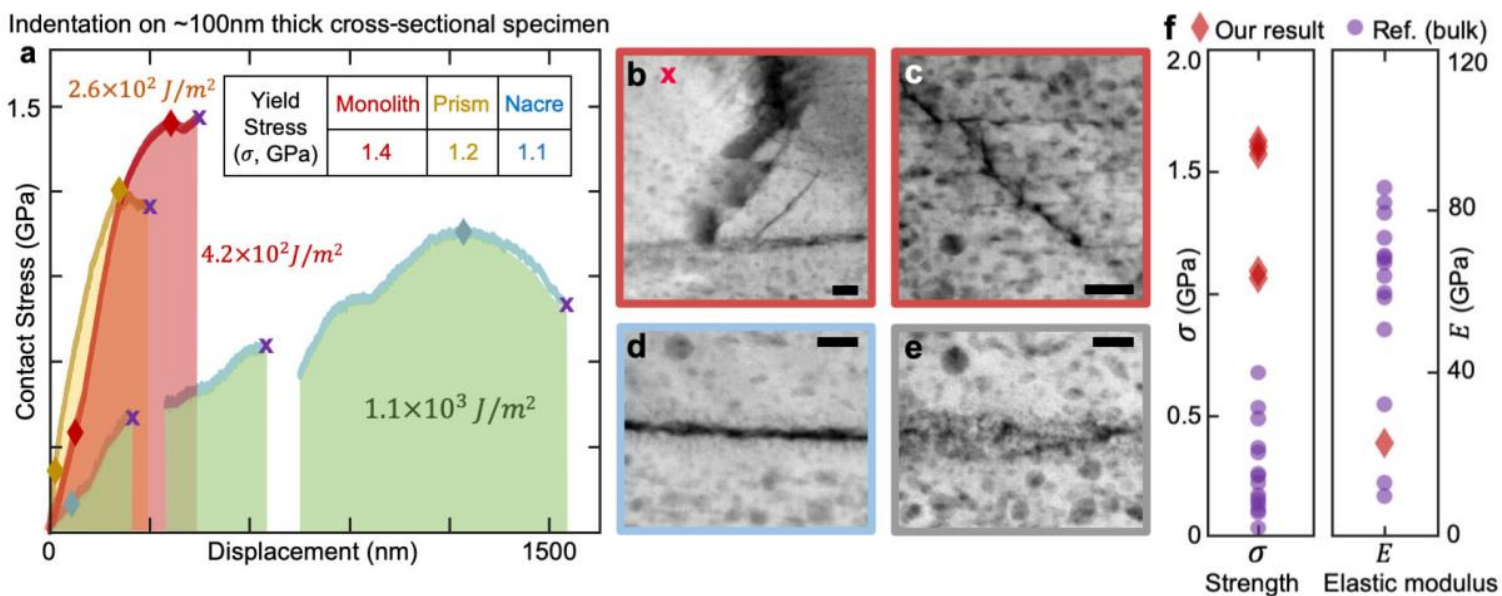

Figure 2. Figure 2. Correlative mechanical response of nacre and crack blunting within and between tablets. a. Correlative compressive contact stress vs. displacement curve showing mechanical response of the nacreous, prismatic, and monolithic region as shown in Figure 1. b. ADF STEM images after the series of the indentation tests showing a crack blunted by an organic boundary. c. ADF STEM image shows crack formed within tablet and blunted by an organic inclusion. d-e. ADF STEM images of Nacre tablet compressed by $0.8 \mathrm{GPa}$ corresponding to the non-linear elastic regime, structure remains fully recoverable - after deformation nacre still preserves both its initial strength and structure. $\mathrm{f}$. Strength and elastic modulus of nacre from contact stress in nanoindentation on the thin cross-sectional specimen in this study and various types of testing - microscale tribology, tensile, compression, and bending- on bulk specimens in previous reports. Scale bar $50 \mathrm{~nm}$.

\section{References}

[1] J. Gim et al, Nature Communications 10, 4822 (2019).

[2] D. C. Hofmann et al, Nature 451 (2008), p.1085-1089.

[3] R. Valiev et al, Nature Materials 3 (2004), p. 511-516.

[4] R. Hovden et al, Nature Communications 6, 10097 (2015).

[5] R. O. Ritchie et al, Nature Materials 10 (2011), p. 817-822.

[6] H. D. Espinosa, J. E. Rim, F. Barthelat. \& M. J. Buehler, Prog. Mater. Sci. 54 (2009), p.1059-1100.

[7] U. G. K. Wegst \& M. F. Ashby, Philos. Mag. 84 (2004), p.2167-2186.

[8] U. Wegst, H. Bai, E. Saiz, A. P. Tomsia \& R. O. Ritchie, Nature Materials 7 (2015), p.672-677

[9] M. A. Meyers, J. McKittrick \& P.-Y. Chen, Science 339 (2013), p.773-779.

[10] M. A. Meyers, P.-Y. Chen, A. Y.-M. Lin \& Y. Seki, Prog. Mater. Sci. 53 (2008), p.1-206.

[11] J. Sun \& B. Bhushan, RSC Advances 2 (2012), p.7617-7632.

[12] A. P. Jackson, J. F. V. Vincent, R. M. Turner \& M. Alexander Robert, Proc. R. Soc. Lond. Ser. B. Biol. Sci. 234 (1988), p.415-440.

[13] F. Barthelat, H. Tang, P. D. Zavattieri, C. M. Li \& H. D. Espinosa, J. Mech. Phys. Solids 55 (2007), p.306-337. 